\title{
Insights into the association of potassium intake with blood pressure: results of a dose-response meta-analysis of randomized controlled trials
}

\author{
Tommaso Filippini ${ }^{1}$, Duarte Torres ${ }^{2,3}$, Carla Lopes ${ }^{2,4}$, Catarina Carvalho ${ }^{2,3}$, Pedro Moreira ${ }^{2,3}$, \\ Androniki Naska ${ }^{5}$, Maria-Iosifina Kasdagli ${ }^{5}$, Marcella Malavolti ${ }^{1}$, Nicola Orsini ${ }^{6}$ and \\ Marco Vinceti ${ }^{1,7}$ \\ ${ }^{1}$ Environmental, Genetic and Nutritional Epidemiology Research Center (CREAGEN), Department of Biomedical, \\ Metabolic and Neural Sciences, University of Modena and Reggio Emilia, Modena, Italy, \\ ${ }^{2}$ EPIUnit - Institute of Public Health, University of Porto, Porto, Portugal, \\ ${ }^{3}$ Faculty of Food and Nutrition Sciences, University of Porto, Porto, Portugal, \\ ${ }^{4}$ Department of Public Health and Forensic Sciences, and Medical Education, Unit of Epidemiology, Faculty of \\ Medicine, University of Porto, Porto, Portugal, \\ ${ }^{5}$ Department of Hygiene, Epidemiology and Medical Statistics, School of Medicine, National and Kapodistrian \\ University of Athens, Athens, Greece, \\ ${ }^{6}$ Department of Public Health Sciences, Karolinska Institute, Stockholm, Sweden and \\ ${ }^{7}$ Department of Epidemiology, Boston University School of Public Health, Boston, USA
}

\begin{abstract}
Introduction: Observational studies provide evidence for an association between potassium intake and BP levels. However, uncertainties still exist about the size and the shape of this relation. Conversely, experimental studies have not been used to estimate dose-response curves, since standard methods can only be applied in trials including at least three exposure groups.

Materials and Methods: We carried out a systematic review of the evidence concerning the effect of potassium supplementation on blood pressure in epidemiologic experimental studies. Following a PubMed search up to June 20, 2019, we included randomized controlled trials (RCTs) encompassing potassium supplementation as the only intervention for at least four weeks. We used a restricted cubic spline model and the 'one-stage' approach to perform a dose-response meta-analysis, a newly-developed statistical procedure which allows inclusion of studies with as few as two categories of exposure (Stat Methods Med Res. 2019;28:1579-1596). Finally, we repeated the analyses stratifying for hypertensive status and use of anti-hypertensive medication.

Results: Overall, we included 33 studies carried out in adult population, with potassium supplementation ranging from 30 to 140 $\mathrm{mmol} /$ day. RCTs' duration ranged from 4 up to 26 weeks. Most of the studies have a cross-over design $(\mathrm{N}=24)$, include hypertensive individuals $(\mathrm{N}=27)$ and subjects not under anti-hypertensive medication $(\mathrm{N}=27)$. Overall, an increase of 40,80 and $120 \mathrm{mmol} / \mathrm{day}$ of potassium resulted in reductions of SBP by -5.64 (95\% CI - 8.78, -2.50), $-4.62(-6.41,-2.84)$ and $-2.54 \mathrm{mmHg}(95 \% \mathrm{CI}-5.14,+0.06)$, respectively. Higher potassium intakes also resulted in reduced DBP levels by -3.57 (95\% CI $-5.55,-1.59),-3.07$ (95\% CI $-5.07,-1.08)$, and $-1.92 \mathrm{mmHg}(95 \% \mathrm{CI}-5.65,1.81)$. The effect of increasing potassium intake on BP was larger among hypertensives than normotensives, and among pharmacologically untreated hypertensives compared to their treated counterparts. Subgroup analyses according to study design (parallel vs. crossover) yielded similar results.
\end{abstract}

Discussion: With the application of advanced dose-response modeling on RCT results, we found a U-shaped relation between potassium intake and blood pressure. A low to moderate increase in potassium intake resulted in a progressive reduction in blood pressure, which was reversed at higher levels of potassium supplementation. The effect was stronger among untreated hypertensives.

Supported by grant GP-EFSA-AFSCO-2017-01 GA09 of the European Food Safety Authority - EFSA. The text reflects only the authors' view; and EFSA is not responsible for any use that may be made of the information it contains.

\section{Conflict of Interest}

There is no conflict of interest 